\title{
Time-domain formulation of cold plasma based on mass-lumped finite elements
}

\author{
W. Tierens and D. De Zutter \\ Electromagnetics Group, Ghent University, Sint-Pietersnieuwstraat 41, Gent, Belgium
}

\begin{abstract}
Recent advances in FDTD simulations of simple dielectrics have opened the possibility of various forms of local refinement [1]. These possibilities are based on writing FDTD as a special case of a finite element technique. We have shown [3] that these techniques can be extended to Body-Of-Revolution (BOR) FDTD which is well-suited for modelling toroidal cavities. Further extending this technique to the time-domain modelling of plasmas presents difficulties: The classical "Whitney" basis-functions (and their analogues in toroidal geometries) are insufficiently smooth to be used as "testing" functions the time-domain constitutive equations of cold plasma [2]. In this paper, we present a set of basis-functions that can be used to write time-domain cold plasma as a mass lumped finite element scheme.
\end{abstract}

Keywords: Cold plasma, Time domain, Finite Element

\section{INTRODUCTION}

The lossless equations describing cold plasma in time domain are [2]

$$
-\frac{\partial \vec{B}}{\partial t}=\vec{\nabla} \times \vec{E}(1 a) \quad \varepsilon_{0} \frac{\partial \vec{E}}{\partial t}=-\sum_{s} \vec{J}_{s}+\mu_{0}^{-1} \vec{\nabla} \times \vec{B}(1 b) \quad \frac{\partial \vec{J}_{s}}{\partial t}=\varepsilon_{0} \omega_{s}^{2} \vec{E}-\vec{\Omega}_{s} \times \vec{J}_{s}(1 c)
$$

We now integrate over a discrete time interval $t-\Delta_{t} / 2 \ldots t+\Delta_{t} / 2$ to obtain:

$$
\varepsilon_{0} \int_{t-\Delta_{t} / 2}^{t+\Delta_{t} / 2} \frac{\partial \vec{E}}{\partial t} d t=-\sum_{s} \int_{t-\Delta_{t} / 2}^{t+\Delta_{t} / 2} \vec{J}_{s} d t+\mu_{0}^{-1} \int_{t-\Delta_{t} / 2}^{t+\Delta_{t} / 2} \vec{\nabla} \times \vec{B} d t
$$

Using eq. 1c and the usual FDTD assumption that the electric field is well approximated by a linear function over a discrete time interval $\Delta_{t}$, we can calculate $\int_{t-\Delta_{t} / 2}^{t+\Delta_{t} / 2} \vec{J}_{S}$ exactly. The discretized solution can be expressed using the $3 \times 3$ matrices $\left[A_{i}\right]$ and $\left[B_{i}\right]$ as

$$
\begin{aligned}
\int_{t-\Delta_{t} / 2}^{t+\Delta_{t} / 2} \vec{J}_{s} d t & =\left[A_{1}\right] \vec{J}_{S}\left(t-\Delta_{t} / 2\right)+\left[A_{2}\right] \vec{E}\left(t-\Delta_{t} / 2\right)+\left[A_{3}\right] \vec{E}\left(t+\Delta_{t} / 2\right) \\
\vec{J}_{S}\left(t+\Delta_{t} / 2\right) & =\left[B_{1}\right] \vec{J}_{S}\left(t-\Delta_{t} / 2\right)+\left[B_{2}\right] \vec{E}\left(t-\Delta_{t} / 2\right)+\left[B_{3}\right] \vec{E}\left(t+\Delta_{t} / 2\right) \\
\vec{E}\left(t+\Delta_{t} / 2\right) & =\left(\varepsilon_{0}+\sum_{s}\left[A_{3}\right]\right)^{-1}\left(-\sum_{s}\left[A_{1}\right] \vec{J}_{S}\left(t-\Delta_{t} / 2\right)+\mu_{0}^{-1} \Delta_{t} \vec{\nabla} \times \vec{B}\right) \\
& +\left(\varepsilon_{0}+\sum_{s}\left[A_{3}\right]\right)^{-1}\left(\varepsilon_{0}-\sum_{s}\left[A_{2}\right]\right) \vec{E}\left(t-\Delta_{t} / 2\right)
\end{aligned}
$$


Explicit expressions for $\left[A_{i}\right]$ and $\left[B_{i}\right]$ can be readily derived but not not be given here. This approach is similar to the implicit approach of [2].

\section{TESTING THE TIME-DOMAIN COLD PLASMA EQUATIONS}

The above time-stepping equations are still continuous in space. They can discretized by writing all electric, magnetic and current components as linear combinations of suitably selected localized basis functions $\vec{E}_{i}, \vec{B}_{i}$ and $\vec{J}_{i}$ :

$$
\vec{E}=\sum_{i} e_{i} \vec{E}_{i} \quad \vec{B}=\sum_{i} b_{i} \vec{B}_{i} \quad \vec{J}_{s}=\sum_{i} j_{s, i} \vec{J}_{i}
$$

Following [1], we use basis functions with the curl inclusion property, i.e. curls of electric field basis functions are exact linear combinations of magnetic field basis functions. This enables us to satisfy Faraday's law (1a) in the strong sense. The timestepping equations for the electric field (1b) and the current (1c) are satisfied weakly by testing both sides of these equations with the electric field basis functions. Just as in simple dielectrics, the term involving $\vec{\nabla} \times \vec{B}$ is problematic because the magnetic basis functions are themselves not curl-conforming. When weighing (4) one typically has to calculate the following integral:

$$
\int \underbrace{\vec{E}_{i} \cdot\left(\varepsilon_{0}+\sum_{s}\left[A_{3}\right]\right)^{-1}}_{f}\left(\vec{\nabla} \times \vec{B}_{j}\right) d V
$$

This integral is still well-defined provided $f$ is curl-conforming. In simple dielectrics, the $\left(\varepsilon_{0}+\sum_{s}\left[A_{3}\right]\right)^{-1}$ factor reduces to a scalar and it hence suffices that the electric basis function $\vec{E}_{i}$ is curl-conforming. However, in plasma's this does no longer suffice and the classical "Whitney" or "Lobatto" basis functions proposed in [1] or their BOR counterparts presented in [3] are not suitable for cold plasma. A suitable extension of these BOR basis functions is given below.

\section{HIGHER ORDER BASIS FUNCTIONS}

Until now we have not given any details on the selection of the proper basis functions except that they must satisfy the curl inclusion property. For e.g. the study of Tokamaks it is convenient to use the BOR FDTD formulation, which decomposes toroidal geometries into toroidal modes. This results in an essentially $2 \mathrm{D}$ scheme where the simulation is performed in a poloidal cross section, In that case the time-stepping equations themselves depend on the toroidal mode number. In general, $n$ th-order basis functions obeying the curl inclusion property in cylindrical coordinates can be constructed as follows: Let $F^{n}(r)$ be a set of functions given by $a+\sum_{0}^{n-1} \frac{c_{n} \ln (r)^{n}}{r}$ and $P^{n}(z)$ be the set of $(n-1)$ th-order polynomials (i.e. polynomials with $n$ coefficients). Let $S=\sin (M \theta)$, 

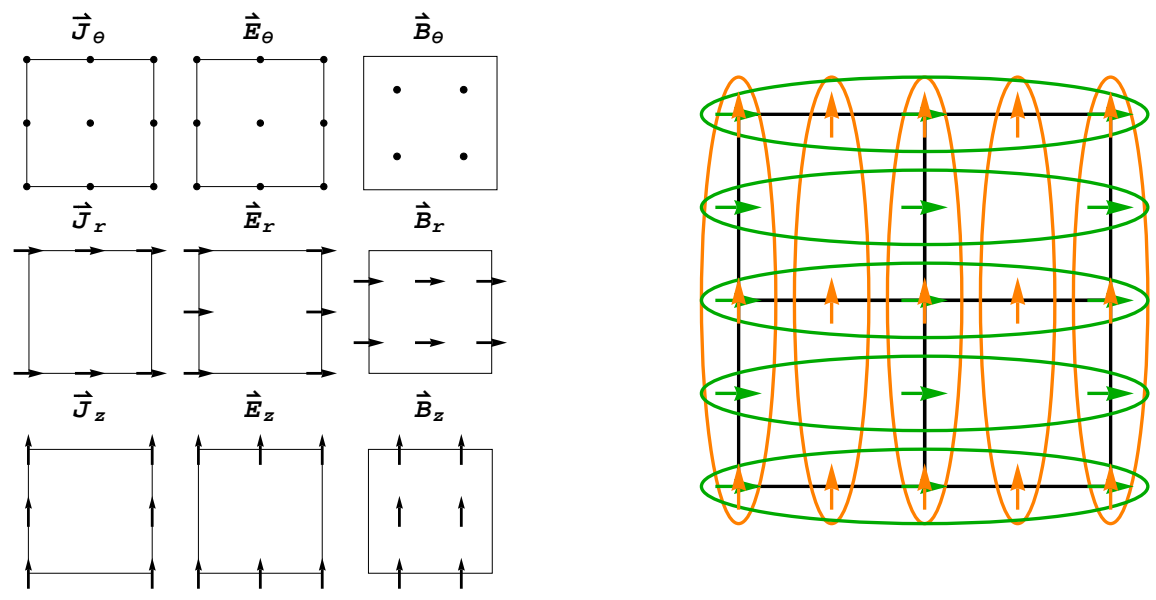

FIGURE 1. Left: Anchor points for the basis-functions on a cell. Right: Block-diagonal structure for $\vec{E}_{z}$ and $\vec{E}_{r}$

$C=\cos (M \theta)$. Elementary electric basis functions are then defined as follows:

$$
E_{\theta} \in F^{n}(r) P^{n}(z) S \quad E_{r} \in F^{n-1}(r) P^{n}(z) C \quad E_{z} \in \int F^{n-1}(r) d r P^{n-1}(z) C
$$

The corresponding elementary magnetic basis functions become

$$
B_{r} \in F^{n}(r) P^{n-1}(z) S \quad B_{\theta} \in F^{n-1}(r) P^{n-1}(z) C \quad B_{z} \in F^{n-1}(r) / r P^{n}(z) S
$$

To discretize space we now introduce elementary cells as depicted in Fig. 1. Each cell extends between $r_{0}$ and $r_{0}+\Delta$ and $z_{0}$ and $z_{0}+\Delta$, and in fact represents an elementary torus as this cell extends over $0 \leq \theta \leq 2 \pi$. For each cell, the dots (top figure, representing $\theta$-components) or arrows (representing radial or z-components) are the so-called anchor points. The actual basis functions that are used to discretize space are defined as linear combinations of the above elementary basis functions in such a way that each contributing elementary basis function is 1 at its anchor point and 0 at all other anchor points. E.g. for $\vec{E}_{\theta}$ this implies that 9 elementary basis functions are combined to get obtain an overall basis function in one particular cell. The functions that are thus defined are natural generalisations of the BOR-FDTD basis-functions introduced in [3] and reduce to those functions when $n=1$. The cell structure (fig. 1) differs from the traditional Yee-like BOR FDTD cell because of the higher smoothness of the basis functions. It is more similar to a second-order "Lobatto" cell [1], but 4 of the $6 \vec{E}_{r}$ and $\vec{E}_{z}$ functions have been moved to the cell corners. The current basis functions are built form the elementary basis functions for the electric field but they have different anchor points (see Fig. 1).

\section{MASS LUMPING}

(BOR) FDTD in simple dielectric media can be derived from finite-element principles by approximating testing integrals such as $(6)$ by trapezoidal integration $[1,3]$. This socalled mass lumping allows to express the effect of place dependent permittivity and 
permeability by means of diagonal matrices which are easily invertible (specifically, $\left[\star_{\varepsilon}\right]_{i, j}=\int \vec{E}_{i} \cdot \vec{E}_{j} d V$ must be inverted). For higher-order cells used here this aproach results in band-diagonal matrices. Low-bandwidth band-diagonal $n \times n$ matrices can be inverted in $O(n)$ time using Cholesky decomposition. This is not explicit but still has the same asymptotic time-complexity as classical FDTD. A detailed analysis shows that the band-diagonal matrices here have an additional block-diagonal structure: there are $O(\sqrt{n})$ band-diagonal blocks whose bandwidth is independent of $n$ (see fig. 1 : the mass-lumping neglects off-diagonal elements between $\vec{R}$-basis-functions in the $z$ direction and between $\vec{z}$-basis-functions in the $R$-direction, leading to blocks whose size is proportional to the width or height of the simulation region, i.e. $O(\sqrt{n})$ ).

\section{NUMERICAL EXAMPLE}

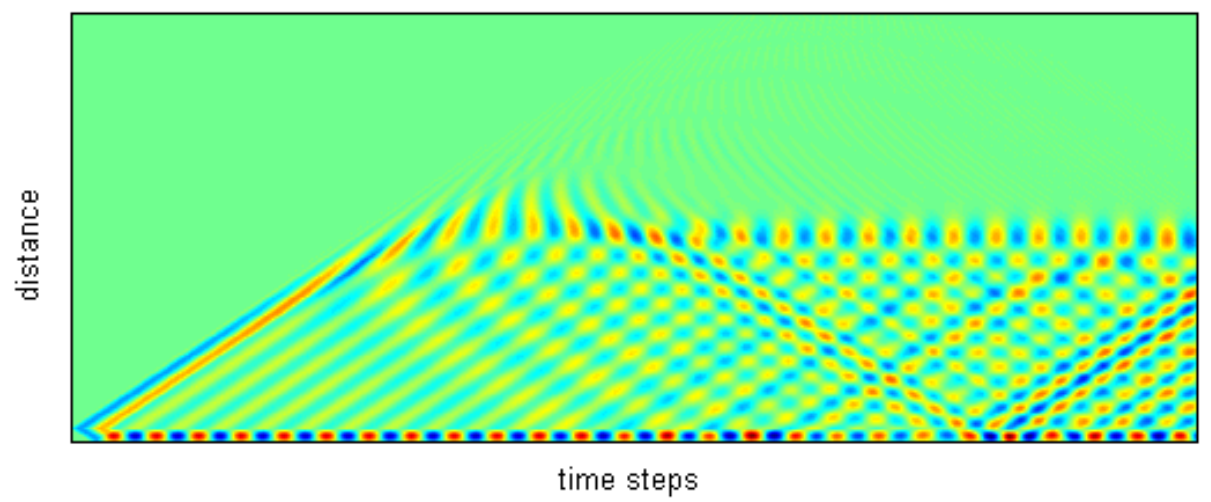

FIGURE 2. Propagation onto a plasma wave beach.

Following [2], we consider a wave incident on a plasma whose density increases as $x^{5}$. The plasma encounters a cutoff condition and is reflected (fig. 2).

\section{CONCLUSION}

An attempt was made to write time-domain cold plasma as a mass lumped finite element method. Unfortunately, we are unable to guarantee the stability of this approach in the magnetized case, and an alternative approach where discretisation in space is performed before discretisation in time seems more promising.

\section{REFERENCES}

1. R. A. Chilton. $H$-, $P$ - and T-refinement strategies for the finite-difference-time-domain (FDTD) method developed via finite-element $(F E)$ principles. PhD thesis, Ohio State University, 2008.

2. D. N. Smithe. Finite-difference time-domain simulation of fusion plasmas at radiofrequency time scales. Physics of Plasmas, 14(14):2537-2549, Apr. 2007.

3. W. Tierens and D. De Zutter. BOR-FDTD subgridding based on finite element principles. Journal of Computational Physics, 2011. 